\title{
Reduced glutathione and Trolox (vitamin E) as extender supplements in cryopreservation of red deer epididymal spermatozoa
}

\author{
Q1 Luis Anel-López a,1 , Manuel Álvarez-Rodríguezb,c,1, Olga García-Álvareza , \\ Mercedes Álvarez b,c Alejandro Maroto-Morales a ${ }^{\text {a }}$ Luis Anel ${ }^{\text {b,c }}$, Paulino de Paz ${ }^{\text {b,d }}$, \\ J. Julián Garde ${ }^{\mathrm{a}}$, Felipe Martínez-Pastor b,d,* \\ a Biology of Reproduction Group. National Wildlife Research Institute (IREC) (UCLM-CSIC-JCCM), Albacete, Spain \\ b ITRA-ULE, INDEGSAL, University of León, 24071 León, Spain \\ c Animal Reproduction and Obstetrics, University of León, 24071 León, Spain \\ d Molecular Biology (Cell Biology), University of León, 24071 León, Spain
}

\section{A R T I C L E I N F O}

\section{Article history:}

Received 26 May 2012

Received in revised form 20 August 2012

Accepted 5 September 2012

Available online xxx

\section{Keywords:}

Epididymal sperm

Red deer

Antioxidant

Trolox

GSH

Cryopreservation

\begin{abstract}
A B S T R A C T
The use of assisted reproductive techniques in cervids is increasing as the commercial use of these species increase. We have tested the suitability of the antioxidants Trolox and reduced glutathione (GSH) for freezing red deer epididymal spermatozoa, aiming at improving postthawing quality. Samples from 19 stags were frozen in a TES-Tris-fructose extender (20\% egg yolk, $8 \%$ glycerol), with 1 or $5 \mathrm{mM}$ of antioxidant. Motility (CASA), lipoperoxidation (malondialdehyde -MDA- production), membrane status, mitochondrial activity, acrosomal status (flow cytometry) and chromatin status (SCSA: \%DFI and \%HDS; flow cytometry) were assessed after thawing and after $6 \mathrm{~h}$ at $39^{\circ} \mathrm{C}$. There were few differences between treatments after thawing, with Trolox reducing MDA production in a dose-response manner. After the incubation, sperm quality decreased and \%DFI increased moderately, with no change for MDA. GSH improved motility, kinematic parameters and mitochondrial status, with a slight increase in \%HDS. GSH $5 \mathrm{mM}$ also increased moderately MDA production and \%DFI, possibly due to enhanced metabolic activity and reducing power. Trolox maintained MDA low, but was detrimental to sperm quality. Trolox might not be appropriate for the cryopreservation of red deer epididymal spermatozoa, at least at the millimolar range. GSH results are promising, especially regarding motility improvement after the post-thawing incubation, and should be selected for future fertility trials.
\end{abstract}

(C) 2012 Published by Elsevier B.V.

\section{Introduction}

The use of artificial reproductive techniques on cervids has become of great interest both for the breeding of farmed species (Asher et al., 1999) and for conservationist purposes (Jabbour et al., 1997; Pukazhenthi and Wildt, 2004). Focusing on spermatozoa collection,

\footnotetext{
* Corresponding author at: INDEGSAL, University of León, 24071, León, Spain. Tel.: +34 687365362.

E-mail address: felipe.martinez@unileon.es (F. Martínez-Pastor).

1 These authors contributed equally to this work.
}

electroejaculation is the obvious choice in farms (Asher et al., 2000). Nevertheless, post-mortem collection has arisen as a convenient source of germplasm, taking advantage of hunting activities (Garde et al., 2006). Spermatozoa collected from harvested males might be applied in deer farms to obtain high quality males for repopulating the hunting reserves. Moreover, since antler size and shape are highly heritable (Kruuk et al., 2002), sperm doses from males with a high-score trophy could become valuable assets for farms.

Most protocols for cryopreserving and using red deer spermatozoa are optimized for semen obtained by electroejaculation (Asher et al., 2000). Consequently, there 
has been an effort to study the differences among electroejaculated and epididymal spermatozoa in this species, which have resulted in extenders adapted to these samples (Fernández-Santos et al., 2006; Fernandez-Santos et al., 2007; Martínez-Pastor et al., 2006; Martínez et al., 2008). As a further step to improve the cryopreservation of red deer epididymal spermatozoa, we have attempted supplementing the extenders with antioxidants, following promising results in other species (Peña et al., 2004; Roca et al., 2005). The mammal spermatozoon might be especially susceptible to oxidative stress (Baker and Aitken, 2004), cryopreservation likely to increase this susceptibility (stressed cells generating free radicals, depletion of antioxidants naturally present in the sample, free radicals from dead spermatozoa). We have shown that the use of some antioxidants could benefit red deer epididymal spermatozoa, both in refrigerated storage (FernandezSantos et al., 2009a), cryopreservation (Fernández-Santos et al., 2007) and post-thawing incubation (DomínguezRebolledo et al., 2010a; Mata-Campuzano et al., in press-b). However, some antioxidant treatments have been unable to improve sperm quality respect to the untreated samples, and some of them have yielded suboptimal or clearly detrimental results. The effect of antioxidants may vary not only depending on concentration (Domínguez-Rebolledo et al., 2010a), but also depending on the species, medium or temperature. For instance, Mara et al. (2005) suggested that refrigerating ram semen in presence of the antioxidant TEMPOL would improve sperm quality and fertility. However, TEMPOL reduced the motility of deer spermatozoa after incubating at $39^{\circ} \mathrm{C}$, although it was able to protect DNA against oxidative stress (Mata-Campuzano et al., in press-b).

In the present study, we have tried to shed some light on the contrasting results ensuing from the application of the antioxidant Trolox on red deer spermatozoa. Trolox is a water-soluble vitamin E analogue with a high capacity to capture free radicals (Mickle and Weisel, 1993), and it is used as standard to check the antioxidant capacity of others molecules (Gavella and Lipovac, 2000). The supplementation of extender with Trolox improved the quality of boar spermatozoa during cooling (Peña et al., 2003) and cryopreservation (Peña et al., 2004), and the quality of stallion semen during refrigerated storage (Ball et al., 2001). Furthermore, we have demonstrated that Trolox at the low millimolar range $(0.1-1 \mathrm{mM})$ is very efficient protecting thawed red deer spermatozoa in presence of oxidative stress, considerably reducing intracellular reactive oxygen species (ROS) and lipid peroxidation, and protecting sperm DNA (Domínguez-Rebolledo et al., 2009, 2010a; MartínezPastor et al., 2008, 2009). However, when this antioxidant was used as a extender supplement in a similar range (3.2 and $6.4 \mathrm{mM}$ ), it was unable to improve sperm quality after thawing (Fernández-Santos et al., 2007). The differences between the application of Trolox to cryopreservation and to post-thaw incubation highlights the need for further investigation, in order to define the real usefulness of this effective antioxidant in deer sperm cryopreservation.

In this study, reduced glutathione (GSH) was examined as an alternative non-enzimatic antioxidant. Glutathione is a major component of the cellular antioxidant system, as a substrate for GSH-transferases and peroxidases (Atmaca, 2004). In addition to its detoxifying function, GSH is able to form disulfide bonds with cysteine residues, regulating the functions of proteins. GSH is depleted during freezing/thawing, possibly due to oxidative stress and cell death (Bilodeau et al., 2000; Gadea et al., 2004), but the outcomes of adding GSH to the freezing extender have been variable. Boar spermatozoa seems to benefit from the supplementation with this antioxidant at 1 and $5 \mathrm{mM}$ (Gadea et al., 2005). In ram semen, Câmara et al. (2011) found no improvement adding GSH $(0.5-2 \mathrm{mM})$ to the cryopreservation extender. Neither did Silva et al. (2011), obtaining even lower motility at $7 \mathrm{mM}$, although GSH improved the ultrastructure of the acrosome at 2 and $5 \mathrm{mM}$. The effects of this antioxidant on deer spermatozoa are still unknown.

In this study, we tested if the supplementation of the freezing extender with Trolox or GSH in the millimolar range ( 1 and $5 \mathrm{mM}$ ) would improve the cryopreservation of red deer epididymal spermatozoa. Our aims were to clarify the results obtained in this species with Trolox, and, for the first time, to test the use of GSH. Ultimately, the objective of this study is the improvement of freezing extenders for red deer, especially those formulated for the cryopreservation of epididymal spermatozoa.

\section{Materials and methods}

\subsection{Reagents and media}

Fluorescence probes YO-PRO-1 and Mitotracker Deep Red were purchased from Invitrogen (Barcelona, Spain), propidium iodide (PI) and PNA-FITC were acquired from Sigma (Madrid, Spain) and acridine orange (chromatographically purified) was purchased from Polysciences (Warrington, PA, USA). Stock solutions of the fluorescence probes were: PI: $7.5 \mathrm{mM}$; PNA-FITC: $0.2 \mathrm{mg} / \mathrm{mL}$; YO-PRO1: $50 \mu \mathrm{M}$; Mitotracker Deep Red: $1 \mathrm{mM}$. All fluorescent stocks were prepared in DMSO - except for PI and PNAFITC, which were prepared in water - and kept at $-20^{\circ} \mathrm{C}$ and in the dark until needed. The stock solution of acridine orange was prepared in water at $1 \mathrm{mg} / \mathrm{mL}$ and kept in the dark at $5^{\circ} \mathrm{C}$. Flow cytometry equipment, software and consumables were purchased from Beckman Coulter (Fullerton, CA, USA) or Becton Dickinson (San Jose, CA, USA). The spectrophotometric assay for malondialdehyde (BIOXYTECH ${ }^{\circledR}$ MDA-586) was purchased from Oxis International (Beverly Hills, CA, USA). The stock solutions of the antioxidants were prepared at $500 \mathrm{mM}$ in DMSO (Trolox) or in water (reduced glutathione, GSH) and stored at $-20^{\circ} \mathrm{C}$. The work medium (TTF380) was composed by $253 \mathrm{mM}$ of TES, $67 \mathrm{mM}$ of Tris and $15 \mathrm{mM}$ of fructose $(380 \mathrm{mOsm} / \mathrm{kg}$, $\mathrm{pH}$ 7.1). The cryopreservation extender was made up by supplementing TTF380 with $20 \%$ of egg yolk, centrifuging $(2500 \times \mathrm{g}$ for $30 \mathrm{~min})$ for removing egg yolk particles, and adding glycerol up to $8 \%$. Solutions for SCSA (Sperm Chromatin Structure Assay) were prepared following Evenson and Jost (2000): TNE buffer (0.01 M Tris- $\mathrm{HCl}, 0.15 \mathrm{M} \mathrm{NaCl}$, $1 \mathrm{mM}$ EDTA, pH 7.4), acid-detergent solution (0.17\% Triton $\mathrm{X}-100,0.15 \mathrm{M} \mathrm{NaCl}, 0.08 \mathrm{~N} \mathrm{HCl}, \mathrm{pH} 1.4)$ and acridine orange solution $\left(0.1 \mathrm{M}\right.$ citric acid, $0.2 \mathrm{M} \mathrm{Na}_{2} \mathrm{HPO}_{4}, 1 \mathrm{mM}$ EDTA, $0.15 \mathrm{M} \mathrm{NaCl}$, pH 6.0; acridine orange was added from 
the stock up to $6 \mu \mathrm{g} / \mathrm{mL}$ ). These solutions were kept at $5^{\circ} \mathrm{C}$ in the dark.

\subsection{Animals and spermatozoa collection}

Samples were obtained from 19 mature stags (Cervus elaphus hispanicus) that were legally hunted in their natural habitat (laws 4/96 from Castilla y León, Spanish Harvest Regulation RD 1201/2005, European Union Regulation 2003/65), in September (during the rut). Game keepers harvested the testes (which were maintained within the scrotum), placed them into plastic bags and kept them at five degrees. Immediately upon arrival at the laboratory, the samples were dissected, collecting spermatozoa from the cauda epididymes by cuts. Before starting sperm collection, the cauda epididymes were cleaned, piercing the superficial blood vessels and wiping out the blood. The concentration of each sample was calculated using a Bürker counting chamber, after diluting the sample in a $2 \%$ glutaraldehyde solution (146 mM glucose, $34 \mathrm{mM}$ sodium citrate tribasic and $24 \mathrm{mM}$ sodium bicarbonate). Just after extraction, an aliquot of each sample was diluted in TTF380, warmed to $37^{\circ} \mathrm{C}$ for $15 \mathrm{~min}$ and evaluated for motility. Only those samples with more than $60 \%$ of motile spermatozoa were used in this experiment. Motility was assessed by CASA (Computer Assisted Sperm Analysis), as described below.

\subsection{Sperm cryopreservation and antioxidant treatments}

Samples from 19 stags were used in the experiment. The cryopreservation extender was divided between 5 tubes. Four of them were supplemented with $1 \mathrm{mM}$ Trolox (T1), $5 \mathrm{mM}$ Trolox (T5), $1 \mathrm{mM}$ GSH (G1) or $5 \mathrm{mM} \mathrm{GSH}$ (G5), respectively, leaving one as the Control extender (C). Each sperm sample was then split between 5 tubes and was slowly diluted with each of the five extenders up to $100 \times 10^{6} \mathrm{~mL}^{-1}$. After one hour of equilibration, the samples were loaded in $0.25-\mathrm{mL}$ French straws. Freezing was carried out using a programmable biofreezer (Kryo 10 Series III; Planer plc., Sunbury-On-Thames, UK) at $-20^{\circ} \mathrm{C} / \mathrm{min}$ down to $-100^{\circ} \mathrm{C}$ and then transferred to liquid nitrogen containers.

Thawing was carried out by dropping the straws in a water bath at $65^{\circ} \mathrm{C}$ for $6 \mathrm{~s}$, and the samples were left $10 \mathrm{~min}$ at ambient temperature $\left(20-22^{\circ} \mathrm{C}\right)$. Samples were analyzed $10 \mathrm{~min}$ post-thawing (post-thawing assessment) and after incubation at $39^{\circ} \mathrm{C}$ for $6 \mathrm{~h}$ in the freezing extender (post-incubation assessment). This incubation temperature was chosen to mimic the internal temperature of a small ruminant female. Sperm samples were assessed for motility (CASA), acrosomal status, membrane integrity, mitochondrial activity, chromatin status and lipoperoxidation, as described below.

\subsection{CASA analysis}

Samples were diluted down to $25 \times 10^{6}$ spermato$\mathrm{zoa} / \mathrm{mL}$ in warm TTF380 and loaded into a Makler counting chamber $\left(10 \mu \mathrm{m}\right.$ depth) at $37^{\circ} \mathrm{C}$. The CASA system consisted of a triocular optical phase contrast microscope
(Eclipse E400; Nikon, Tokyo, Japan), equipped with a warming stage at $38^{\circ} \mathrm{C}$ and a Basler A312fc digital camera (Basler Vision Technologies, Ahrensburg, Germany). The camera was connected to a computer by an IEEE 1394 interface. Images were captured and analyzed using the ISAS software v. 1.2 (Proiser, Valencia, Spain). Sampling was carried out using a $\times 10$ negative phase contrast objective (no intermediate magnification). Image sequences were saved and analyzed afterwards. The standard parameter settings were: 50 frames/s; $20-90 \mu \mathrm{m}^{2}$ for head area; $\mathrm{VCL}>10 \mu \mathrm{m} / \mathrm{s}$ to classify a spermatozoon as motile. For each spermatozoa, the software rendered the percentage of motile spermatozoa, three velocity parameters (VCL: velocity according to the actual path; VSL: velocity according to the straight path; VAP: velocity according to the smoothed path), three track linearity parameters (LIN: linearity; STR: straightness: WOB: wobble), the ALH (amplitude of the lateral displacement of the sperm head), the BCF (head beat-cross frequency), total motility, defined as the proportion of spermatozoa with VCL $>10 \mu \mathrm{m} / \mathrm{s}$, and progressive motility as the proportion of spermatozoa with VCL $>25 \mu \mathrm{m} / \mathrm{s}$ and STR $>80 \%$.

2.5. Evaluation of sperm viability, acrosomal status, mitochondrial activity and apoptotic markers

Several physiological traits were assessed by using fluorescent probes and flow cytometry, which have been previously described for red deer (Martínez-Pastor et al., 2006, 2008). Samples were diluted down to $10^{6} \mathrm{~mL}^{-1}$ in TTF380, and stained using the fluorophore combinations PI/PNA-FITC for studying viability and acrosomal status, and YO-PRO-1/PI/Mitotracker deep red for studying membrane permeability and mitochondrial status. PNA-FITC was used at $100 \mu \mathrm{g} / \mathrm{mL}$, PI at $6 \mu \mathrm{M}$, YO-PRO- 1 at $0.1 \mu \mathrm{M}$ and Mitotracker Deep Red at $0.1 \mu \mathrm{M}$. In all cases, Hoescht 33342 was added at $5 \mathrm{mM}$, in order to discriminate debris. Spermatozoa stained in these two solutions were incubated for $15 \mathrm{~min}$ in the dark before being analyzed by flow cytometry.

The sperm populations showed in this paper were: YO-PRO-1 negative (considered viable spermatozoa), YO-PRO-1+/PI- (spermatozoa with increased plasma membrane permeability, considered compromised membranes), Mitotracker deep red positive (viable spermatozoa with active mitochondria) and PNA positive (spermatozoa with damaged acrosomes).

\subsection{Sperm chromatin structure assay}

Chromatin stability was assessed following the SCSA, as performed previously with epididymal red deer samples (Martínez-Pastor et al., 2009). Acridine orange (AO) fluorescence shifts from green (dsDNA) to red (ssDNA) depending on the degree of DNA denaturation. Samples were diluted in TNE buffer to a final sperm concentration of $2 \times 10^{6}$ cells $/ \mathrm{mL}$, and stored at $-80^{\circ} \mathrm{C}$. For analysis, the samples were thawed on crushed ice and submitted to acid-induced denaturation of DNA in situ and staining with acridine orange. A volume of $200 \mu \mathrm{L}$ of sample was pipetted in a flow cytometry tube, and it was immediately 
mixed with $0.4 \mathrm{~mL}$ of the acid-detergent solution. After $30 \mathrm{~s}, 1.2 \mathrm{~mL}$ of the acridine orange solution was added to the tube. The tube was kept on ice 3 min before flow cytometry analysis.

\subsection{Flow cytometry analyses}

Flow cytometry analyses were carried out with a CyAn ADP flow cytometer (Beckman Coulter, Brea, CA, USA), with semiconductor lasers emitting at $405 \mathrm{~nm}$ (violet; Hoechst 33342), $488 \mathrm{~nm}$ (blue; YO-PRO-1, FITC, PI, acridine orange), and $635 \mathrm{~nm}$ (red; Mitotracker Deep Red). Filters used for each fluorochrome were 450/50 (blue) for Hoechst 33342, 530/40 (green) for YO-PRO-1 and FITC, $613 / 20$ (red) for PI, and 665/20 for Mitotracker deep red. The system and event analyses were controlled using the Summit software provided with the cytometer. All the parameters were read using logarithmic amplification. For each sample, 5000 spermatozoa were recorded, saving the data in flow cytometry standard (FCS) v. 3 files. The analysis of the flow cytometry data was carried out using WEASEL v. 3 (WEHI, Melbourne, Australia). The PI/PNA-FITC and YO-PRO-1/PI/Mitotracker deep red combinations were analyzed as previously described for red deer (Martínez-Pastor et al., 2006, 2008).

For the analysis of SCSA samples Evenson and Jost (2000), we used a FACScalibur flow cytometer (Becton Dickinson) and the acquisition software CellQuest v. 3. We analyzed 5000 events per sample, exciting the acridine orange with the Ar-ion $488 \mathrm{~nm}$ laser and using a 530/30 filter for the green fluorescence of DNAds-bound $\mathrm{AO}$, and a 650 long pass filter for the red fluorescence of DNAss-bound AO. Data was saved in saving the data in flow cytometry standard (FCS) v. 2 files, which were processed using the $\mathrm{R}$ statistical environment. We calculated the DNA Fragmentation Index (DFI) for each spermatozoa as the ratio of red fluorescence respect to total fluorescence (red + green). From the DFI values we obtained the percentage of spermatozoa with high fragmentation index (\%DFI, DFI $>25 \%$ ), and the percentage of spermatozoa with high DNA sustainability (HDS), defined as those events with green fluorescence above channel 600.

\subsection{Assessment of lipid peroxidation}

The susceptibility of the spermatozoa to lipoperoxidation was assessed using the Bioxitech ${ }^{\circledR}$ MDA-586 kit (Oxis International, Foster, CA, USA) to detect malondialdehyde (MDA) concentration (Domínguez-Rebolledo et al., 2010a). To induce MDA release, samples were previously diluted with PBS to $10^{7} \mathrm{~mL}^{-1}$ and incubated for 30 min at $37^{\circ} \mathrm{C}$ with $40 \mu \mathrm{M}$ of $\mathrm{FeSO}_{4}$ and $200 \mu \mathrm{M}$ sodium ascorbate. The sample was then mixed with the reactive provided in the kit and incubated at $45^{\circ} \mathrm{C}$ for $1 \mathrm{~h}$. The tubes were centrifuged and the supernatant was transferred to wells (200 $\mu \mathrm{L} /$ well) in a 96-well flat bottom transparent plate (Nunc, Roskilde, Denmark). The plate absorbance at $586 \mathrm{~nm}$ was read on a multipurpose microplate reader (Synergy HT, BIO-TEK, Winooski, Vermont, USA). The MDA production (nmol of
MDA per 1( matozoa) was calculated from a standard curve gene...... irom know quantities of MDA.

\subsection{Statistical analysis}

Data were analyzed in the $\mathrm{R}$ statistical environment ( $R$ Development Core Team, 2011). The effects of the antioxidant supplements were analyzed using linear mixed-effects models, with incubation time, antioxidant and antioxidant concentration in the fixed part of the models, and including the male as the grouping factor in the random part of the models. Results are presented as mean \pm SEM, or effect sizes (mean \pm SEM of the respective coefficients) respect to the control (that is, the variation of the variable submitted to a given treatment, relative to the control).

\section{Results}

The results were clearly affected by the sampling endpoint, with treatment effects being significant after the incubation. Just after thawing, the motility of sperm samples was similar across treatments, not showing many differences between the Control and the antioxidant treatments (Fig. 1). Despite obtaining a good total motility after thawing $(60.2 \% \pm 3.2$; sub Fig. $1 \mathrm{a})$, the progressive motility of the samples was very low (sub Fig. 1b), with only $4.4 \% \pm 0.3$ of the spermatozoa being identified as progressive (post-thawing overall average).

After incubating the samples for $6 \mathrm{~h}$, the motility was still acceptable, decreasing significantly (Control: $46.4 \% \pm 5.3$ ). Velocity did not change significantly, and variables defining track shape (LIN and WOB, sub Fig. 1d and e) only decreased slightly. Both GSH concentrations kept MOT similar to the post-thawing Control $(P>0.05)$, while increasing VCL (increase respect to the post-thawing Control of $12.7 \pm 3.7$ for $1 \mathrm{mM}, P=0.002$, and $22.1 \pm 3.7$ for $5 \mathrm{mM}, P<0.001$; sub Fig. 1c); VAP and VSL were also higher for both GSH-treated samples after the incubation (VAP: increase of $9.8 \pm 2.2$ for $1 \mathrm{mM}$ and $17.5 \pm 2.2$ for $5 \mathrm{mM}$, $P<0.001$; VSL: increase of $3.5 \pm 0.8$ for $5 \mathrm{mM}, P<0.001$ ). WOB was also significantly increased by GSH (sub Fig. 1e). WOB is the ratio between VAP (velocity according to the smoothed path) and VCL (curvilinear velocity), and its increase indicates a less sinuous trajectory. ALH also increased with GSH (sub Fig. 1f), indicating a more "energetic" movement. Contrarily, Trolox depressed motility, both in quantity (total motility) and in quality (lower velocity, WOB and ALH). Although this effect was slightly more pronounced at $5 \mathrm{mM}$, both concentrations produced the same effect.

Similarly to motility, the status of sperm organelles was little affected by the antioxidants in the post-thawing assessment, but several differences arose during the incubation (Fig. 2). Sperm viability (intact plasma membrane capable to keep both YO-PRO-1 and PI out of the cell; sub Fig. 2a) did not vary significantly between treatments either after thawing or after the incubation. However, the proportion of the subpopulation with altered plasma membrane permeability (PI- but not being able to prevent YO-PRO-1 inflow; sub Fig. 2b) was significantly lower for 
(a) Total motility

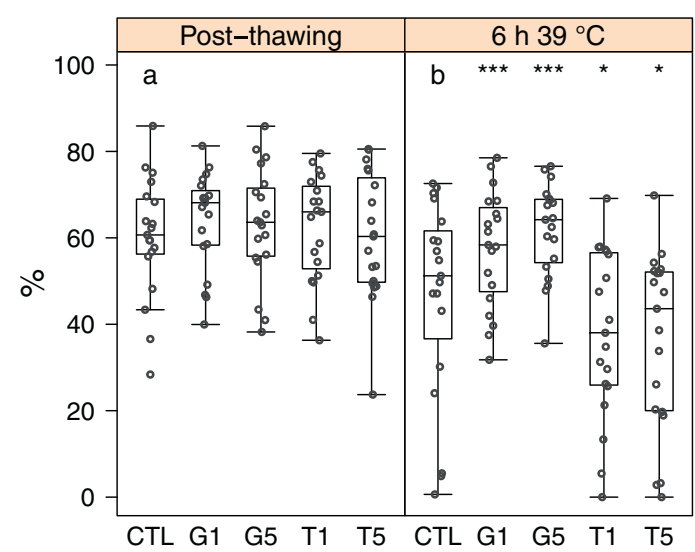

(c) VCL (curvilinear velocity)

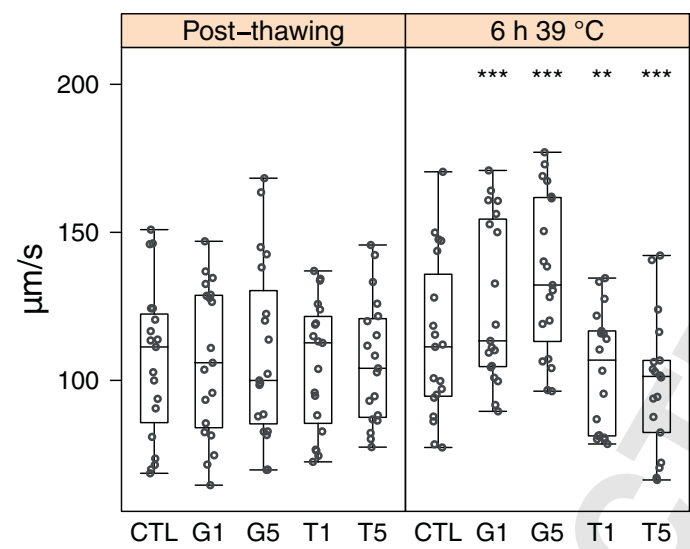

(e) WOB (wobble)

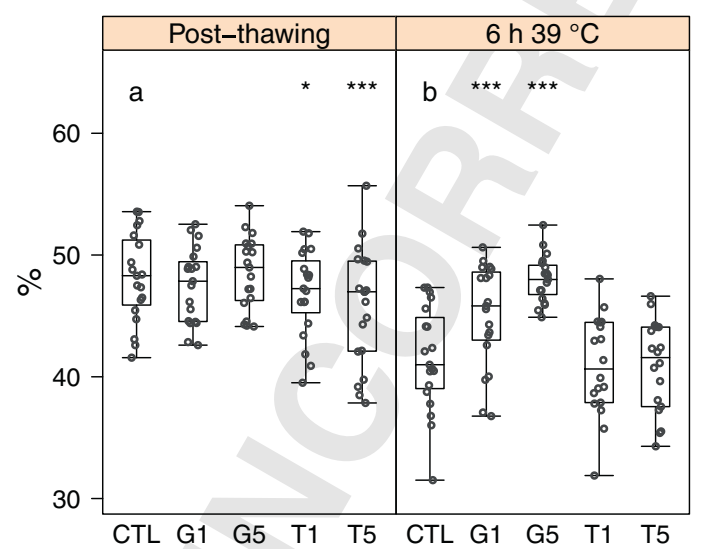

(b) Progressive motility

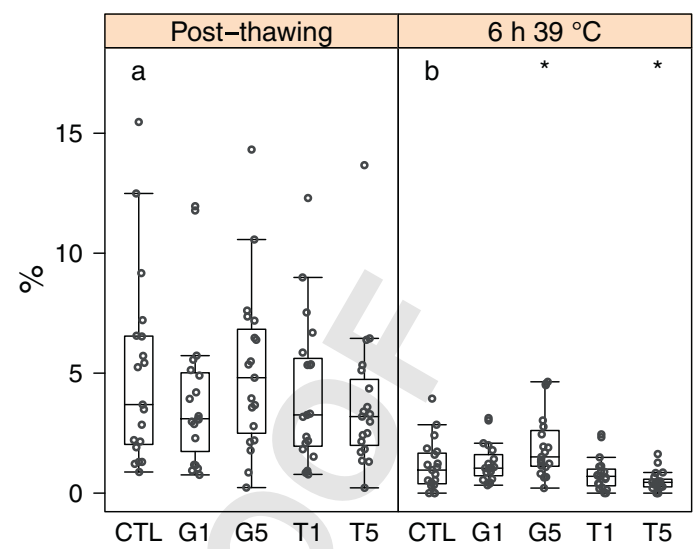

(d) LIN (linearity)

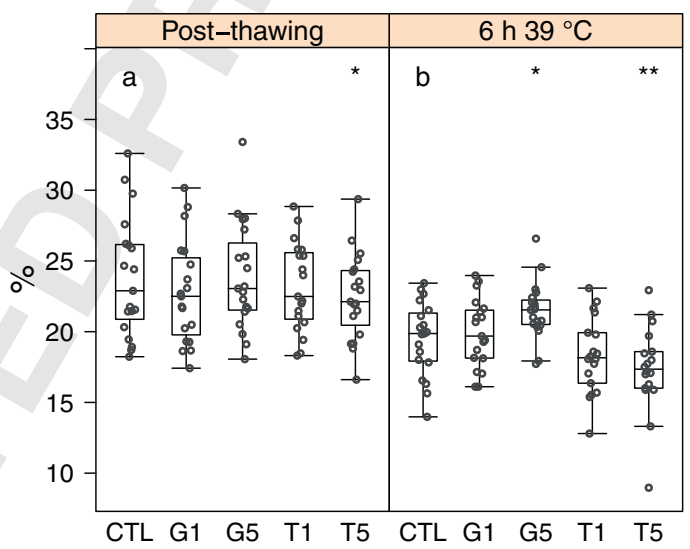

(f) ALH (lateral head displacement)

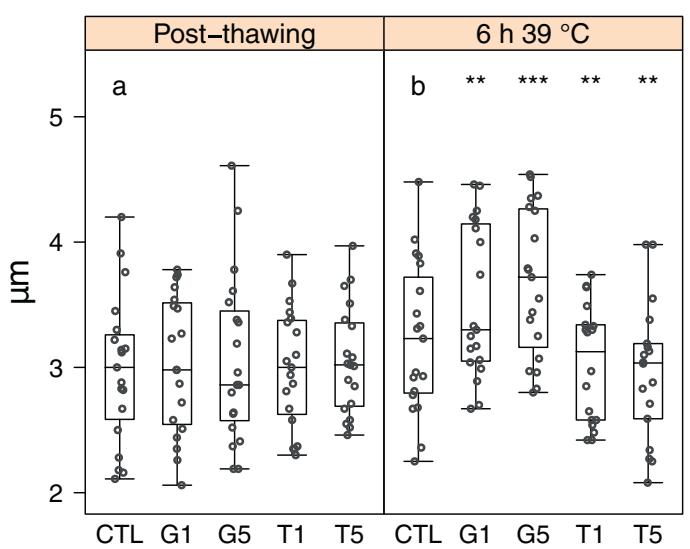

Fig. 1. Effects of antioxidant treatments on red deer sperm motility at post-thawing or after $6 \mathrm{~h}$ of incubation at $39{ }^{\circ} \mathrm{C}$. Dots show the actual values for each treatment (CTL: Control; G1: GSH $1 \mathrm{mM}$; G5: GSH 5 mM; T1: Trolox $1 \mathrm{mM}$; T5: Trolox 5 mM). The superimposed boxes span from the 1st to the 3rd quartile, with the inner line showing the median, and whiskers span up to the extreme observations within 1.5 times the interquartile range. Letters on the top show significant differences between the control samples of both endpoints (different letters indicate $P<0.05$ ), whereas asterisks indicate a significant effect of the antioxidant treatments respect to the control value within each endpoint $\left({ }^{*} P<0.05 ;{ }^{* *} P<0.01 ;{ }^{* * *} P<0.001\right)$. 
(a) Viability (YO-PRO-1-)

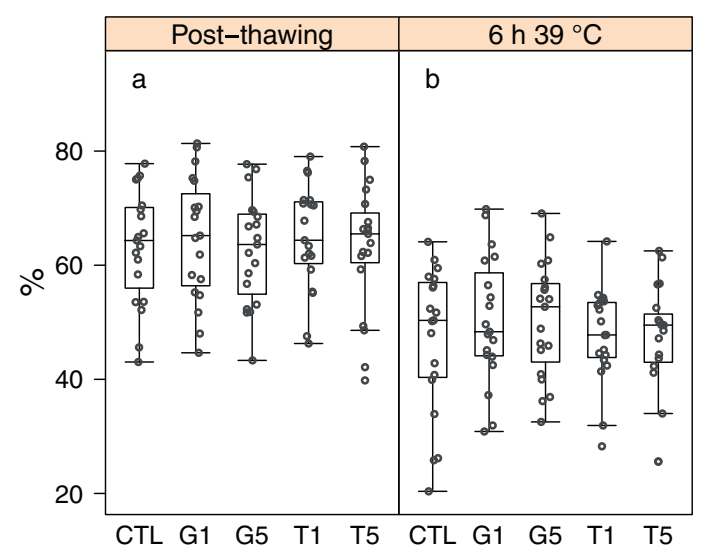

(c) Active mitochondria (Mitotracker+)

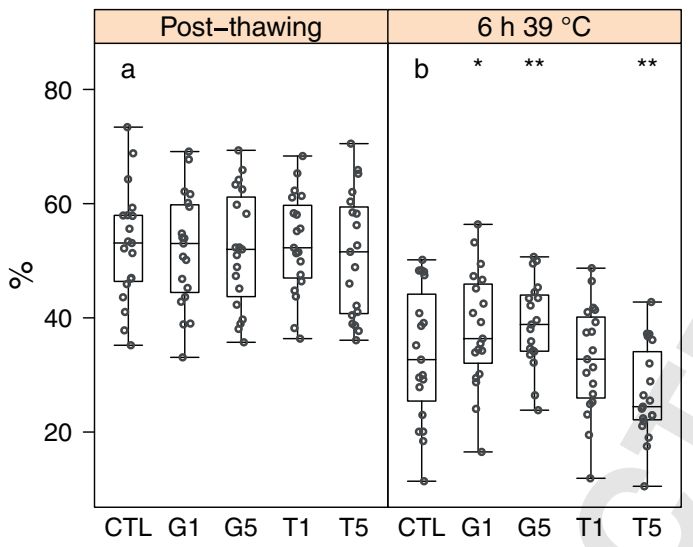

(b) Compromised membrane (YO-PRO-1+/PI-)

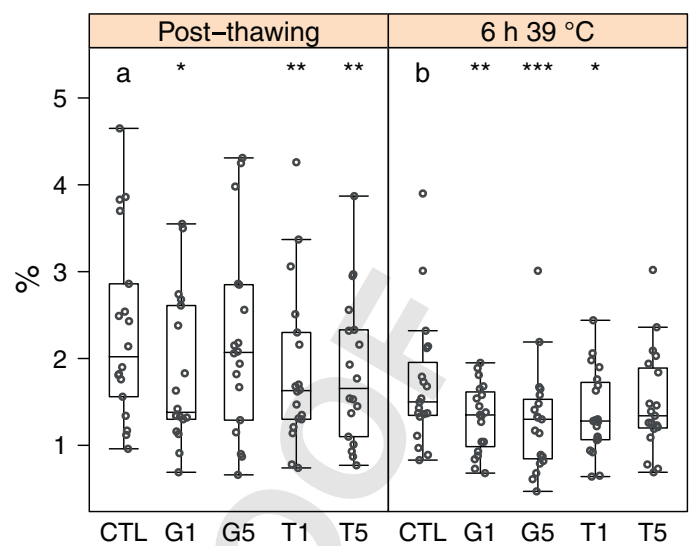

(d) Damaged acrosomes (PNA+)

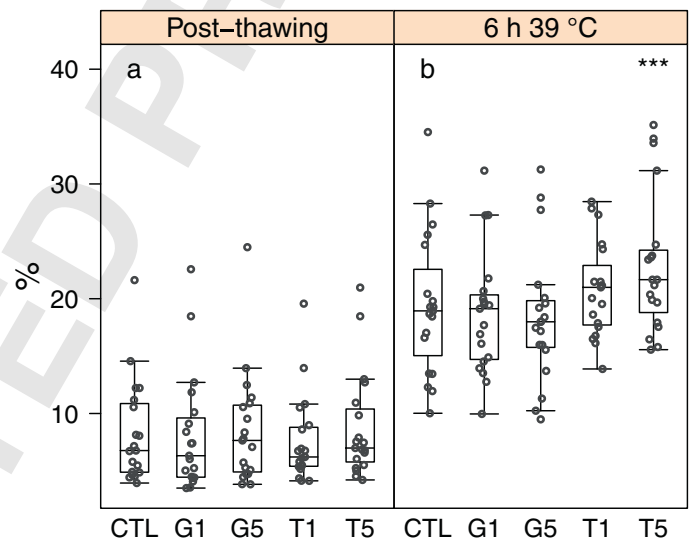

Fig. 2. Effects of antioxidant treatments on red deer sperm physiology at post-thawing or after $6 \mathrm{~h}$ of incubation at $39{ }^{\circ} \mathrm{C}$, assessed by using fluorescent probes and flow cytometry. Figure elements are described in the caption of Fig. 1.

GSH $1 \mathrm{mM}$ and both Trolox treatments just after thawing (nevertheless, the proportion of this subpopulation was very small). The proportion of this population was even lower after the incubation (below $2 \%$ for most samples; $P<0.01$ comparing with the post-thawing endpoint), possibly due to cell death. Both GSH concentrations and Trolox $1 \mathrm{mM}$ significantly decreased this population compared to the Control.

The incubation caused an overall decrease of $18.1 \pm 1.2$ percent points in the proportion of spermatozoa with active mitochondria (sub Fig. 2c). Both GSH concentrations moderated this decrease to $14.2 \pm 2.1$ percent points $(P<0.05)$, while it was more pronounced for Trolox $5 \mathrm{mM}$ $(25.9 \pm 2.4$ percent points; $P<0.01)$. This negative effect of Trolox $5 \mathrm{mM}$ was also observed in the proportion of spermatozoa with damaged acrosomes according to PNA stain (sub Fig. 2d). Acrosomal damage increased 11.1 \pm 1.1 percent points after the incubation (Control samples, $P<0.001$ ), with this increase being significantly larger in the samples treated with Trolox $5 \mathrm{mM}(14.7 \pm 1.1$ percent points, $P<0.001$ ).
However, Trolox prevented lipid peroxidation in a dosedependent manner (sub Fig. 3a). No differences were detected between the post-thawing and post-incubation analyses regarding malondialdehyde production, but Trolox $1 \mathrm{mM}$ reduced it by $3.8 \pm 0.4 \mathrm{nmol}$ per $10^{8}$ cells and Trolox $5 \mathrm{mM}$ by $7.9 \pm 0.4 \mathrm{nmol}$ per $10^{8}$ cells (overall for both endpoints, $P<0.001)$. GSH had no effect on this parameter, except for GSH $5 \mathrm{mM}$ after the incubation, which induced a small increase of $1.8 \pm 0.7 \mathrm{nmol}$ per $10^{8}$ cells $(P=0.007)$.

The analysis of DNA status by SCSA showed that only a small proportion of spermatozoa presented altered chromatin (\%DFI, sub Fig. 3b). This proportion significantly increased after the incubation, although remaining within acceptable \%DFI levels (overall median of $11.7 \%$ ), with only a few samples above $20 \%$ of \%DFI. At this endpoint, samples frozen with GSH $5 \mathrm{mM}$ showed a higher sensitivity to chromatin alterations (increase of $5.8 \pm 2.2$ percent points respect to the Control, $P=0.011$ ). This effect was also noted in the \%HDS parameter (sub Fig. 3c), which was higher after the incubation in samples frozen with GSH (increasing $4.2 \pm 0.7$ for $1 \mathrm{mM}$ and $7.3 \pm 0.7$ for $5 \mathrm{mM}$, comparing to 
(a) Lipoperoxidation (MDA production)

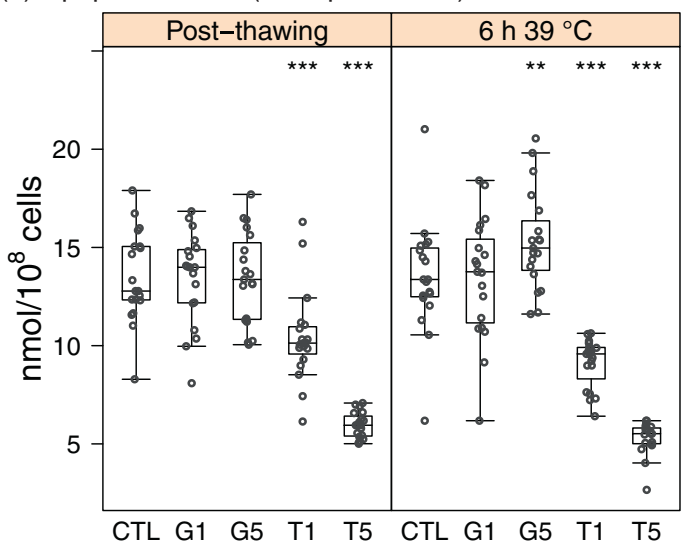

(b) DNA damage (SCSA, \%DFI)

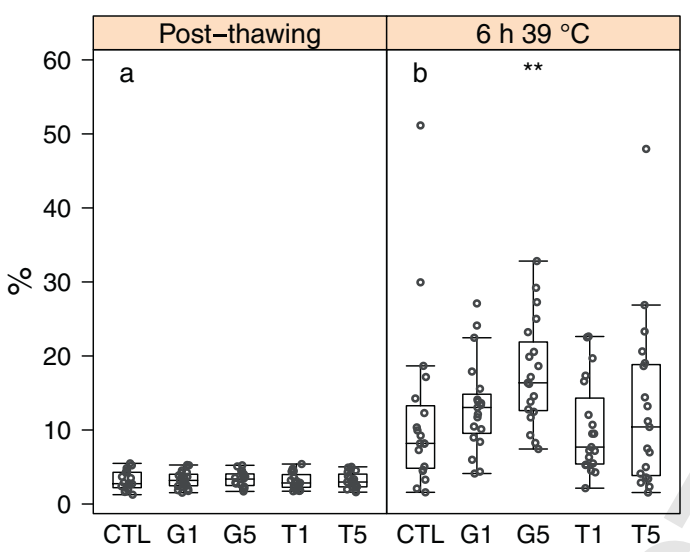

(c) DNA stainability (SCSA, \%HDS)

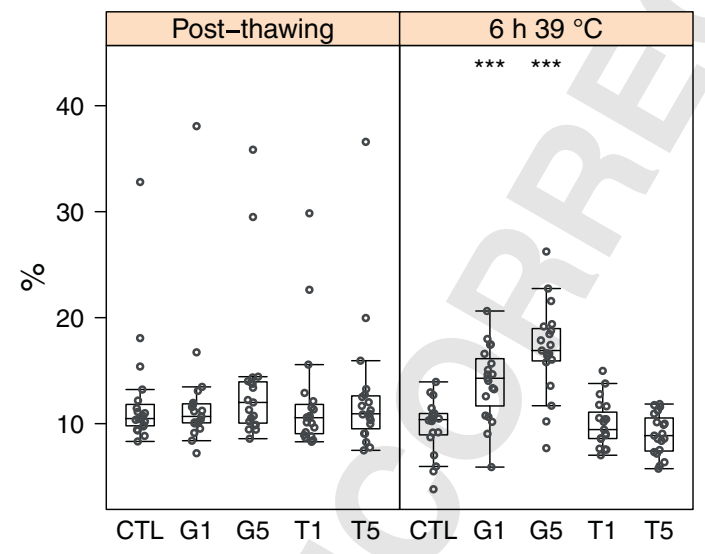

Fig. 3. Effects of antioxidant treatments on the lipoperoxidation and chromatin status of red deer sperm at post-thawing or after $6 \mathrm{~h}$ of incubation at $39^{\circ} \mathrm{C}$. Figure elements are described in the caption.

the Control; $P<0.001)$. Neither incubation nor Trolox had a significant effect in \%HDS.

\section{Discussion}

This study has produced two important results, the first that the antioxidants GSH and Trolox affected the outcome of red deer epididymal spermatozoa cryopreservation and the second, that these effects were masked in the postthawing analysis, but clearly seen after several hours at physiological temperature. These results give some indication of the effects of antioxidants in sperm physiology during the cryopreservation process.

Submitting spermatozoa to cooling and freezing/thawing may induce the production of free radicals, which might add to or worsen the effects of cold shock and cryopreservation (Chatterjee and Gagnon, 2001). Spermatozoa are highly sensitive to lipid peroxidation, but this process can be prevented or reverted by many antioxidant mechanisms present in the cell or in the seminal plasma (Storey, 1997). In this study, we have worked with epididymal spermatozoa from red deer, as a valuable and convenient source of germplasm from hunting reserves (Garde et al., 2006). Epididymal spermatozoa are protected by a range of antioxidant enzymes (Vernet et al., 2004), but the samples are highly diluted upon collection, reducing protection to that provided by the sperm extender. Moreover, we have observed that part of the spermatozoa lose the cytoplasmic droplet during the cryopreservation process (unpublished data), which is likely to reduce its resistance to the oxidative stress (Nichi et al., 2007).

In order to improve the quality of deer epididymal spermatozoa, Trolox appears as an attractive option. We have reported that the application of Trolox to thawed deer epididymal spermatozoa at $100 \mu \mathrm{M}$ slightly benefited sperm quality (Martínez-Pastor et al., 2008), where it showed a high free radical scavenging activity, even at only $10 \mu \mathrm{M}$ (Martínez-Pastor et al., 2009). Trolox at $1 \mathrm{mM}$ greatly diminished the susceptibility of deer spermatozoa to oxidative stress after thawing and washing (DomínguezRebolledo et al., 2009). Similar experiments with thawed spermatozoa compared several antioxidants, and Trolox (at 0.1 and $1 \mathrm{mM}$ ) was one of the most efficient preventing intracellular ROS rise, lipid peroxidation and DNA damage (Domínguez-Rebolledo et al., 2010a). Interestingly no inhibitory effect of Trolox on sperm motility was identified.

The present study shows that Trolox, at least at the low millimolar range, might not be appropriate for freezing epididymal deer spermatozoa, which agrees with a previous study (Fernández-Santos et al., 2007), using higher concentrations ( 3.2 and $6.4 \mathrm{mM}$ ) and a different methodology for freezing and analyzing the samples. Other authors have found positive results cryopreserving boar semen with Trolox at 100 and $200 \mu \mathrm{M}$, finding a protective effect in sperm membranes that depended on the semen fraction frozen (Peña et al., 2004). These same authors found a dose-dependent protective effect of Trolox on sperm motility and mitochondrial activity (Peña et al., 2003), both at 30 and $120 \mathrm{~min}$ after thawing. These results contrast with those obtained in deer, since we found a decrease in motility and mitochondrial activity. It is possible that Trolox could exert a negative effect through an excessive scavenging activity, since ROS have a physiological function, participating in intracellular signaling, and being associated with many important events in the spermatozoa (Aitken, 1995; O'Flaherty et al., 2006).

However, the ROS scavenging activity alone do not explain Trolox effects. The antioxidants TEMPOL or rutin, 
which are excellent ROS scavengers too, produced motility inhibition when incubating ram and red deer spermatozoa post-thawing (Mata-Campuzano et al., in press-a; in press-b), but this did not occur when using Trolox (Domínguez-Rebolledo et al., 2010a). It is possible that Trolox could interfere with intracellular pathways during critical steps of the freezing/thawing procedure, an effect that could be uncovered during the post-thawing incubation (when Trolox might exert little negative effects by itself).

In contrast, GSH improved the quality of red deer spermatozoa after cryopreservation and incubation. The protection of mitochondrial activity during the incubation is specially important, given the role of this organelle not only in maintaining the energetic status of the spermatozoa, but also in pathways regulating motility, capacitation and apoptosis (Peña et al., 2009). Although the presence of a high proportion of spermatozoa with active mitochondria might not be essential for higher fertility (Al Naib et al., 2011), the GSH-treated samples showed a concomitant increase not only in the proportion of motile spermatozoa, but also in velocity and ALH. This increase in motility might represent a difference in fertility if these samples were used for artificial insemination, given the importance of sperm velocity in deer fertility (Malo et al., 2005). However, results in other ruminants have not been so encouraging. Foote et al. (2002) cryopreserved bull semen with $0.5 \mathrm{mM}$ GSH, reporting some improvement in motility only after $12 \mathrm{~h}$ of incubation, which was significant only when adding $100 \mathrm{U} / \mathrm{mL}$ of superoxide dismutase together with the GSH. These authors conducted a field trial using GSH at 0.5 and $1 \mathrm{mM}$ in extended semen (not cryopreserved), not observing an improvement on fertility. Tuncer et al. (2010), cryopreserving bull semen with 0.5 and $2 \mathrm{mM} \mathrm{GSH}$, did not obtain any improvement in sperm motility or other quality parameters just after thawing. Only DNA damage was significantly lower with $0.5 \mathrm{mM}$, but it was not accompanied by higher fertility. Similarly, studies on ram semen have shown few positive results when supplementing the cryopreservation extender with GSH. For example, Bucak et al. (2008) (using GSH $5 \mathrm{mM}$ ) reported an improvement in the antioxidant status in the thawed samples, but similar results for sperm quality and lipid peroxidation. Câmara et al. (2011) reported that the total antioxidant activity and sperm quality post-thawing was similar among treatments (GSH at 0.5, 1 and $2 \mathrm{mM}$ ), while Silva et al. (2011) found that GSH at $7 \mathrm{mM}$ had negative effects on ram semen motility and mitochondrial and acrosomal ultrastructure. Nevertheless, 2 and 5 mM GSH improved acrosomal ultraestructure, but with no improvements in motility, viability, acrosomal integrity or mitochondrial status.

When studying lipid peroxidation, we have found that the supplementation with GSH increased the production of MDA after the incubation. We must point out that lipid peroxidation did not increase during the incubation in the Control samples, suggesting that deer epididymal spermatozoa are able to cope with oxidative stress after thawing in an acceptable manner (although we cannot discard a protective effect of the freezing extender). This is an interesting observation, since the antioxidant capacity of frozen/thawed spermatozoa seems to decrease considerably (Bilodeau et al., 2000). Indeed, other studies have suggested that deer spermatozoa might be little prone to lipoperoxidation during post-thawing incubation, unless external oxidants are added (Domínguez-Rebolledo et al., 2010a,b). The increase of MDA in samples supplemented with GSH at $5 \mathrm{mM}$ may be related to the increased metabolic activity, given the higher motility and mitochondrial activity of GSH-treated samples after the incubation. Similarly, Tuncer et al. (2010) observed an increase of MDA production after cryopreservation of bull semen with GSH at 0.5 and $2 \mathrm{mM}$. Nevertheless, it is important to keep in mind that the increasing MDA levels in our samples frozen with GSH $5 \mathrm{mM}$ was not linked with a negative effect on motility or sperm physiology, and they could be well below dangerous levels. On the contrary, Trolox decreased MDA production efficiently (agreeing to previous studies (Domínguez-Rebolledo et al., 2010a)). The lower values after thawing indicate that the effect of Trolox on MDA production was exerted during the freezing/thawing process, and not during the incubation. These results support our hypothesis that the effects of Trolox (positive and negative) that we have observed in this study are due to its activity during the freezing/thawing process, rather than during the post-thawing incubation.

DNA damage was very low after thawing, similar to results in fresh deer epididymal spermatozoa (Garcia-Macias et al., 2006). The chromatin of red deer spermatozoa, as assessed by SCSA, seems to be very resilient to damage even after long periods of storage without antioxidants (Fernandez-Santos et al., 2009a,b). After the incubation, most samples remained with low levels of chromatin alterations, agreeing with previous studies on cervids (Fernandez-Santos et al., 2009b; Gosálvez et al., 2011). Trolox had no effect after the incubation, but it might be due to the already low levels of chromatin alterations. We have reported in other studies a protective effect of Trolox on sperm DNA, but this effect was only noticeable in samples treated with oxidants and other stressing situations (Domínguez-Rebolledo et al., 2009, 2010a).

Regarding GSH, we observed an increase in \%DFI and \%HDS, especially when using it at $5 \mathrm{mM}$. Garcia-Macias et al. (2006) demonstrated in samples from several species that spermatozoa obtained from the caput epididymis presented a higher \%DFI and \%HDS than those obtained from the cauda. These results were compatible with a lower chromatin condensation in the caput, rather than with DNA damage, as confirmed by other authors studying the condensation of sperm chromatin during epididymal transit (Golan et al., 1996). We believe that this is the case in our study, and that the reducing power provided by GSH might be used by nuclear peroxidases to break the disulfide bonds, especially in non-viable spermatozoa, with broken membranes. Nevertheless, these results must be taken into account if fertility trials are undertaken using spermatozoa frozen in the presence of GSH.

\section{Conclusions}

This study presents GSH as a possible supplement for the cryopreservation of red deer epididymal spermatozoa. However, it is necessary to perform fertility trials (in vitro 
and in vivo), in order to test if the post-incubation results reflect improved fertility, although the post-incubation results are promising. Trolox is a powerful antioxidant that has performed well when added to deer spermatozoa after thawing and washing, but it does not seem suitable as a supplement for the cryopreservation extender. Nevertheless, future studies could consider the use of Trolox at lower concentrations, and testing possible synergistic effects with other antioxidants.

It is important to highlight that this is the first study testing the effect of GSH as an extender supplement for freezing red deer spermatozoa. If our findings result in improved fertility, GSH supplementation might represent a feasible option for improving the results of AI using frozen epididymal spermatozoa in this species.

\section{Acknowledgements}

This work has been supported by Junta de Castilla y León (LE019A10-2), by the Spanish Ministry of Science and Innovation (AGL2010-15758/GAN), and by CDTI (Centro para el desarrollo tecnológico industrial, IDI-20080478). L. Anel-López was supported by a Ph.D. grant (JCCM). Manuel Álvarez-Rodríguez was supported by a FPI grant (Spanish Ministry of Science and Innovation). F. Martínez-Pastor was supported by the Ramón y Cajal program (RYC-200802560, Ministry of Science and Innovation, Spain).

\section{References}

Aitken, R.J., 1995. Free radicals, lipid peroxidation and sperm function. Reprod. Fertil. Dev. 7, 659-668.

Al Naib, A., Hanrahan, J.P., Lonergan, P., Fair, S., 2011. In vitro assessment of sperm from bulls of high and low field fertility. Theriogenology 76 , 161-167.

Asher, G.W., Monfort, S.L., Wemmer, C., 1999. Comparative reproductive function in cervids: implications for management of farm and zoo populations. J. Reprod. Fertil. Suppl. 54, 143-156.

Asher, G.W., Berg, D.K., Evans, G., 2000. Storage of semen and artificial insemination in deer. Anim. Reprod. Sci. 62, 195-211.

Atmaca, G., 2004. Antioxidant effects of sulfur-containing amino acids. Yonsei Med. J. 45, 776-788.

Baker, M.A., Aitken, R.J., 2004. The importance of redox regulated pathways in sperm cell biology. Mol. Cell. Endocrinol. 216, 47-54.

Ball, B.A., Medina, V., Gravance, C.G., Baumbe, J., 2001. Effect of antioxidants on preservation of motility, viability and acrosomal integrity of equine spermatozoa during storage at 5 degrees $C$. Theriogenology 56 , 577-589.

Bilodeau, J.F., Chatterjee, S., Sirard, M.A., Gagnon, C., 2000. Levels of antioxidant defenses are decreased in bovine spermatozoa after a cycle of freezing and thawing. Mol. Reprod. Dev. 55, 282-288.

Bucak, M.N., Ateșşahin, A., Yüce, A., 2008. Effect of anti-oxidants and oxidative stress parameters on ram semen after the freeze-thawing process. Small Rumin. Res. 75, 128-134.

Câmara, D.R., Silva, S.V., Almeida, F.C., Nunes, J.F., Guerra, M.M.P., 2011. Effects of antioxidants and duration of pre-freezing equilibration on frozen-thawed ram semen. Theriogenology 76, 342-350.

Chatterjee, S., Gagnon, C., 2001. Production of reactive oxygen species by spermatozoa undergoing cooling, freezing, and thawing. Mol. Reprod. Dev. 59, 451-458.

Domínguez-Rebolledo, A.E., Fernández-Santos, M.R., García-Alvarez, O., Maroto-Morales, A., Garde, J.J., Martínez-Pastor, F., 2009. Washing increases the susceptibility to exogenous oxidative stress in red deer spermatozoa. Theriogenology 72, 1073-1084.

Domínguez-Rebolledo, Á.E., Fernández-Santos, M.R., Bisbal, A., RosSantaella, J.L., Ramón, M., Carmona, M., Martínez-Pastor, F., Garde, J.J., 2010a. Improving the effect of incubation and oxidative stress on thawed spermatozoa from red deer by using different antioxidant treatments. Reprod. Fertil. Dev. 22, 856-870.
Domínguez-Rebolledo, Á.E., Martínez-Pastor, F., Fernández-Santos, M.R., del Olmo, E., Bisbal, A., Ros-Santaella, J.L., Garde, J.J., 2010. Comparison of the TBARS assay and BODIPY C11 probes for assessing lipid peroxidation in red deer spermatozoa. Reprod Domest Anim 45, e360-e368.

Evenson, D., Jost, L., 2000. Sperm chromatin structure assay is useful for fertility assessment. Methods Cell Sci. 22, 169-189.

Fernandez-Santos, M.R., Martínez-Pastor, F., Garcia-Macias, V., Esteso, M.C., Soler, A.J., de Paz, P., Anel, L., Garde, J.J., 2007. Extender osmolality and sugar supplementation exert a complex effect on the cryopreservation of Iberian red deer (Cervus elaphus hispanicus) epididymal spermatozoa. Theriogenology 67, 738-753.

Fernandez-Santos, M., Dominguez-Rebolledo, A., Esteso, M., Garde, J., Martínez-Pastor, F., 2009a. Refrigerated storage of red deer epididymal spermatozoa in the epididymis, diluted and with vitamin C supplementation. Reprod. Domest. Anim. 44, 212-220.

Fernandez-Santos, M.R., Martínez-Pastor, F., Matias, D., DominguezRebolledo, A.E., Esteso, M.C., Montoro, V., Garde, J.J., 2009. Effects of long-term chilled storage of red deer epididymides on DNA integrity and motility of thawed spermatozoa. Anim. Reprod. Sci. 111, 93-104.

Fernández-Santos, M.R., Esteso, M.C., Montoro, V., Soler, A.J., Garde, J.J., 2006. Cryopreservation of Iberian red deer (Cervus elaphus hispanicus) epididymal spermatozoa: effects of egg yolk, glycerol and cooling rate. Theriogenology 66, 1931-1942.

Fernández-Santos, M.R., Martínez-Pastor, F., García-Macías, V., Esteso, M.C., Soler, A.J., Paz, P., Anel, L., Garde, J.J., 2007. Sperm characteristics and DNA integrity of Iberian red deer (Cervus elaphus hispanicus) epididymal spermatozoa frozen in the presence of enzymatic and nonenzymatic antioxidants. J. Androl. 28, 294-305.

Foote, R.H., Brockett, C.C., Kaproth, M.T., 2002. Motility and fertility of bull sperm in whole milk extender containing antioxidants. Anim. Reprod. Sci. 71, 13-23.

Gadea, J., Sellés, E., Marco, M.A., Coy, P., Matás, C., Romar, R., Ruiz, S., 2004. Decrease in glutathione content in boar sperm after cryopreservation. Effect of the addition of reduced glutathione to the freezing and thawing extenders. Theriogenology 62, 690-701.

Gadea, J., Garcia-Vazquez, F., Matas, C., Gardon, J.C., Canovas, S., Gumbao, D., 2005. Cooling and freezing of boar spermatozoa: supplementation of the freezing media with reduced glutathione preserves sperm function. J. Androl. 26, 396-404.

Garcia-Macias, V., Martínez-Pastor, F., Alvarez, M., Garde, J.J., Anel, E., Anel, L., de Paz, P., 2006. Assessment of chromatin status (SCSA) in epididy$\mathrm{mal}$ and ejaculated sperm in Iberian red deer, ram and domestic dog. Theriogenology 66, 1921-1930.

Garde, J.J., Martínez-Pastor, F., Gomendio, M., Malo, A.F., Soler, A.J., Fernandez-Santos, M., Esteso, M.C., Garcia, A.J., Anel, L., Roldan, E.R.S., 2006. The application of reproductive technologies to natural populations of red deer. Reprod. Domest. Anim. 2 (Suppl. 41), 93-102.

Gavella, M., Lipovac, V., 2000. Antioxidative effect of melatonin on human spermatozoa. Arch. Androl. 44, 23-27.

Golan, R., Cooper, T.G., Oschry, Y., Oberpenning, F., Schulze, H., Shochat, L., Lewin, L.M., 1996. Changes in chromatin condensation of human spermatozoa during epididymal transit as determined by flow cytometry. Hum. Reprod. 11, 1457-1462.

Gosálvez, J., López-Fernández, C., Fernández, J.L., Gouraud, A., Holt, W.V. 2011. Relationships between the dynamics of iatrogenic DNA damage and genomic design in mammalian spermatozoa from eleven species. Mol. Reprod. Dev. 78, 951-961.

Jabbour, H.N., Hayssen, V., Bruford, M.W., 1997. Conservation of deer: contributions from molecular biology, evolutionary ecology, and reproductive physiology. J. Zool. 243, 461-484.

Kruuk, E.B., Slate, J., Pemberton, J.M., Brotherstone, S., Guinness, F., Clutton-Brock, T., 2002. Antler size in red deer: heritability and selection but no evolution. Evolution 56, 1683-1695.

Malo, A.F., Garde, J.J., Soler, A.J., García, A.J., Gomendio, M., Roldan, E.R.S. 2005. Male fertility in natural populations of red deer is determined by sperm velocity and the proportion of normal spermatozoa. Biol. Reprod. 72, 822-829.

Mara, L., Accardo, C., Pilichi, S., Dattena, M., Chessa, F., Chessa, B., Branca, A., Cappai, P., 2005. Benefits of TEMPOL on ram semen motility and in vitro fertility: a preliminary study. Theriogenology 63, 2243-2253.

Martínez, A.F., Martínez-Pastor, F., Alvarez, M., Fernández-Santos, M.R., Esteso, M.C., de Paz, P., Garde, J.J., Anel, L., 2008. Sperm parameters on Iberian red deer: electroejaculation and post-mortem collection. Theriogenology 70, 216-226.

Martínez-Pastor, F., Martínez, F., Garcia-Macias, V., Esteso, M., Anel, E. Fernandez-Santos, M., Soler, A., de Paz, P., Garde, J., Anel, L., 2006. A pilot study on post-thawing quality of Iberian red deer spermatozoa (epididymal and electroejaculated) depending on glycerol concentration and extender osmolality. Theriogenology 66, 1165-1172.

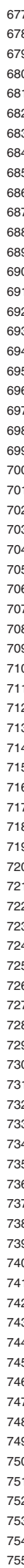


Martínez-Pastor, F., Fernandez-Santos, M.R., del Olmo, E., DominguezRebolledo, A.E., Esteso, M.C., Montoro, V., Garde, J.J., 2008. Mitochondrial activity and forward scatter vary in necrotic, apoptotic and membrane-intact spermatozoan subpopulations. Reprod. Fertil. Dev. 20,547-556.

Martínez-Pastor, F., Aisen, E., Fernández-Santos, M.R., Esteso, M.C., Maroto-Morales, A., García-Alvarez, O., Garde, J.J., 2009. Reactive oxygen species generators affect quality parameters and apoptosis markers differently in red deer spermatozoa. Reproduction 137, 225-235.

Mata-Campuzano, M., Alvarez-Rodríguez, M., Alvarez, M., Anel, L., de Paz, P., Garde, J., Martínez-Pastor, F. Effect of several antioxidants on thawed ram spermatozoa submitted to $37^{\circ} \mathrm{C}$ up to four hours. Reprod. Domest. Anim. in press, http://dx.doi.org/10.1111/ j.1439-0531.2012.01990.x

Mata-Campuzano, M., Alvarez-Rodríguez, M., del Olmo, E., FernándezSantos, M., Garde, J.J., Martínez-Pastor, F. Quality, oxidative markers and DNA fragmentation of red deer thawed spermatozoa after incubation at $37^{\circ} \mathrm{C}$ in presence of several antioxidants. Theriogenology in press, http://dx.doi.org/10.1016/j.theriogenology.2011.12.018

Mickle, D.A., Weisel, R.D. 1993. Future directions of vitamin E and its analogues in minimizing myocardial ischemia-reperfusion injury and its analogues in minimizing myocardial ischemia-reperfusion injury. Can. J. Cardiol. 9, 89-93.

Nichi, M., Goovaerts, I.G.F., Cortada, C.N.M., Barnabe, V.H., De Clercq, J.B.P., Bols, P.E.J., 2007. Roles of lipid peroxidation and cytoplasmic droplets on in vitro fertilization capacity of sperm collected from bovine epididymides stored at 4 and $34^{\circ} \mathrm{C}$. Theriogenology 67, 334-340.

O'Flaherty, C., de Lamirande, E., Gagnon, C., 2006. Positive role of reactive oxygen species in mammalian sperm capacitation: triggering and modulation of phosphorylation events. Free Radic. Biol. Med. 41, 528-540.

Peña, F.J., Johannisson, A., Wallgren, M., Rodriguez Martínez, H., 2003. Antioxidant supplementation in vitro improves boar sperm motility and mitochondrial membrane potential after cryopreservation of different fractions of the ejaculate. Anim. Reprod. Sci. 78, 85-98.

Peña, F.J., Johannisson, A., Wallgren, M., Rodriguez Martínez, H., 2004. Antioxidant supplementation of boar spermatozoa from different fractions of the ejaculate improves cryopreservation: changes in sperm membrane lipid architecture. Zygote 12, 117-124.

Peña, F.J., Rodríguez Martínez, H., Tapia, J.A., Ortega Ferrusola, C., González Fernández, L., Macías García, B., 2009. Mitochondria in mammalian sperm physiology and pathology: a review. Reprod. Domest. Anim. 44, 345-349.

Pukazhenthi, B.S., Wildt, D.E., 2004. Which reproductive technologies are most relevant to studying, managing and conserving wildlife? Reprod. Fertil. Dev. 16, 33-46.

Development Core Team, R., 2011. R: A Language and Environment for Statistical Computing. R Foundation for Statistical Computing, Vienna, Austria.

Roca, J., Rodríguez, M.J., Gil, M.A., Carvajal, G., Garcia, E.M., Cuello, C., Vazquez, J.M., Martínez, E.A., 2005. Survival and in vitro fertility of boar spermatozoa frozen in the presence of superoxide dismutase and/or catalase. J. Androl. 26, 15-24.

Silva, S.V., Soares, A.T., Batista, A.M., Almeida, F.C., Nunes, J.F., Peixoto, C.A., Guerra, M.M.P., 2011. In vitro and in vivo evaluation of ram sperm frozen in tris egg-yolk and supplemented with superoxide dismutase and reduced glutathione. Reprod. Domest. Anim. 46, 874-881.

Storey, B.T., 1997. Biochemistry of the induction and prevention of lipoperoxidative damage in human spermatozoa. Mol. Hum. Reprod. 3, 203-213.

Tuncer, P.B., Bucak, M.N., Büyükleblebici, S., Sarı̈zzkan, S., Yeni, D., Eken, A., Akalın, P.P., Kinet, H., Avdatek, F., Fidan, A.F., Gündoğan, M., 2010. The effect of cysteine and glutathione on sperm and oxidative stress parameters of post-thawed bull semen. Cryobiology 61, 303-307.

Vernet, P., Aitken, R.J., Drevet, J.R., 2004. Antioxidant strategies in the epididymis. Mol. Cell. Endocrinol. 216, 31-39. 